\title{
Thermal stratification effects on flow over a generic urban canopy
}

\author{
3 V.B.L. Boppana $\cdot$ Z-T Xie · I.P. Castro
}

5 Received: date / Accepted: date

\begin{abstract}
The influence of local surface heating and cooling on flow over urbanlike roughness is investigated using large-eddy simulations (LES). By adjusting the incoming or outgoing heat flux from the ground surface, various degrees of local thermal stratification, represented by a Richardson number $\left(R i_{\tau}\right)$, were attained. Drag and heat transfer coefficients, turbulence structure, integral length scales, and the strength of quadrant events that contribute to momentum and heat fluxes were obtained and are compared with locally stable, neutral and unstable flows. With increasing $R i_{\tau}$, or equivalently as the flow characteristics change from local thermal instability to stability, a gradual decline in the drag and heat transfer coefficients is observed. These values are found to be fairly independent of the type of thermal boundary condition (constant heat flux or constant temperature) and domain size. The maps of anisotropy invariants showed that for the values of $R i_{\tau}$ considered, turbulence structures are almost the same in shape for neutral and unstable cases but differ slightly from those in the stable case. The degree of anisotropy is found to decrease as $R i_{\tau}$ increases from -2 to 2.5. Compared to the neutral case, the integral length scales are shortened in the streamwise and vertical direction by ground cooling, but enhanced in the vertical direction with ground heating. Quadrant analysis showed that increase in floor heating increases the strength of ejections above the canopy. However, the contributions of updrafts or downdrafts to heat flux are found not to be significantly influenced by the type of local thermal stratification for the values of $R i_{\tau}$ considered. The transport mechanisms of momentum and heat above the canopy are found to be very similar in both locally unstable and stable flows.
\end{abstract}

Keywords Correlations · Drag coefficient · Heat transfer coefficient · Quadrant events · Turbulent structures

V. B. L. Boppana · Zheng-Tong Xie · Ian P. Castro

Facultu of Engineering and Environment

University of Southampton

Tel: $+44(0) 2380592320$

Fax: $+44(0) 2380593058$

E-mail: V.B.Boppana@ soton.ac.uk 
31

\section{Introduction}

Do the effects of thermal stratification have a dominant role on the structure of turbulence and mechanisms of pollutant transport in and above roughness canopies of various morphologies? To investigate this, numerous field, wind-tunnel and computational studies have been conducted, especially in the last two to three decades. The field studies included several vegetation (e.g. Gao et al., 1989) and urban (e.g. Christen et al., 2007) areas to understand the similarities and differences in the transport of momentum and heat over the two kinds of canopies. One of the similarities that was observed is that sweep events contribute most to the momentum flux below and immediately above the canopy height and ejection events dominate further above the canopy; these events are considered to be the signatures of the large coherent structures. Li and Bou-Zeid (2011) discussed in detail the dissimilarity of momentum, temperature and water vapour transport with increasing instability from measurements over a vineyard and a lake. However, it is difficult to obtain comprehensive, spatially detailed measurements from the field owing to instrument limitations and the impossibility of obtaining repeated and controlled conditions; wind-tunnel and computational studies can therefore be particularly useful.

The simplest geometry, yet challenging if thermal stratification is included, is two-dimensional (2-D) street canyons. Allegrini et al. (2013), Huizhi et al. (2003), Kovar-Panskus et al. (2002), for example, have studied such cases in wind tunnels and shown that surface heating greatly influences the number and intensity of vortices within the canyon. Similar observations have also been made from various computational studies (e.g. Cai, 2012; Kim and Baik, 1999; Park et al., 2012). In the case of 3 -D roughness morphologies, by adjusting the temperatures of the approach flow and the floor of a wind-tunnel, Uehara et al. (2000) created a thermally stratified atmospheric boundary layer over square arrays of roughness obstacles. They showed that a stable atmosphere results in weak cavity eddies whilst unstable conditions enhances the strength of cavity eddies. Using LES, Inagaki et al. (2012) simulated a complete day time atmospheric boundary layer over a square array of cubes with ground and roof heating and showed that the turbulent organized structures above the canopy are correlated to the strong upward motion that occurs within the cavity of the arrays. All these 'generic' urban canopy investigations clearly imply that the dispersion of pollutants might be affected by surface heating. Computational studies on field sites like DAPPLE (Dispersion of Air Pollution and its Penetration into the Local Environment) have certainly suggested that weak unstable conditions in the approach flow have notable effects on scalar dispersion (Xie et al., 2013).

It is necessary to quantify the effects of such thermal stratification on street and/or neighborhood scale flows in order to provide required parameters for city or regional scale modelling. For this purpose, we first performed computations to simulate passive scalar dispersion from a surface area source in an array of uniform and random height blocks (Boppana et al., 2010), followed by simulation of heat transfer from the strongly heated leeward surface of a large building (Boppana et al., 2013). These computations showed good agreement with the wind-tunnel experiments of Pascheke et al. (2008) and Richards et al. (2006) respectively. The former LES study had no buoyancy and the latter included its effects on the surrounding flow. These previous 
investigations led naturally to the current LES study where, instead of heating a single surface of an isolated obstacle, the entire ground surface (i.e. all streets, in direct contact with the atmosphere) is uniformly heated (see Fig. 1) or cooled and the resulting buoyancy effects are included to model the flow over an array of staggered cubes. It is to be noted that, in this study, thermal stratification in a fully-developed boundary layer is a result of surface heating or cooling within the bottom canopy, which is rather different to the case of a thermally stratified approach flow over an unheated region (e.g. Xie et al., 2013).

The overall goal of the present paper is to obtain insights on the effects of uniform ground heating or cooling on the flow over an array of uniform height staggered buildings. To address this, the following objectives were formulated: (1) to quantify the effects of thermal stratification on the surrounding flow, including the turbulence structure, and (2) to determine the similarities and/or differences in momentum and heat transport for stable, neutral and unstable stratified flows via assessment of the affects of stratification on surface drag and heat transfer coefficients. We present the numerical description in Sect. 2, followed by the results and conclusions in Secs. 3 and 4 respectively.

\section{Numerical Details and Settings}

The filtered continuity and Navier-Stokes equations governing unsteady incompressible flow are

$$
\frac{\partial u_{i}}{\partial x_{i}}=0
$$

95 and,

$$
\frac{\partial u_{i}}{\partial t}+\frac{\partial u_{i} u_{j}}{\partial x_{j}}=-\frac{1}{\rho}\left(\frac{\partial p}{\partial x_{i}}+\delta_{i 1} \frac{\partial\langle P\rangle}{\partial x_{1}}\right)+\frac{\partial}{\partial x_{j}}\left(\frac{\tau_{i j}}{\rho}+v \frac{\partial u_{i}}{\partial x_{j}}\right)+f \delta_{i 3} .
$$

The resolved-scale velocity and pressure are respectively given by $u_{i}$ and $p$ with $u$, $v$ and $w$ the streamwise, lateral and vertical velocity components respectively. The flow was driven by a constant mean streamwise pressure gradient $\partial\langle P\rangle / \partial x$ and $\delta_{i 1}$ is the Kronecker-delta. $f \delta_{i 3}$ is the body force due to thermal buoyancy and is estimated using the Boussinesq approximation. $\rho$ and $v$ are the density and kinematic viscosity of the fluid. $\tau_{i j}$ is the subgrid-scale (SGS) Reynolds stress and was handled using the Smagorinsky model in conjunction with a Lilly damping function near the walls. We set Smagorinsky's constant $C_{s}=0.1$ since this was found to provide satisfactory results in our earlier computations (Boppana et al., 2010).

In the streamwise $(x)$ and lateral $(y)$ directions, periodic boundary conditions were employed. Stress free conditions were imposed on the top of the domain, i.e.,

$$
\frac{\partial u}{\partial z}=\frac{\partial v}{\partial z}=0 ; \quad w=0
$$

No slip conditions were set on the bottom surface $(z=0)$ and on all faces of the roughness elements. 


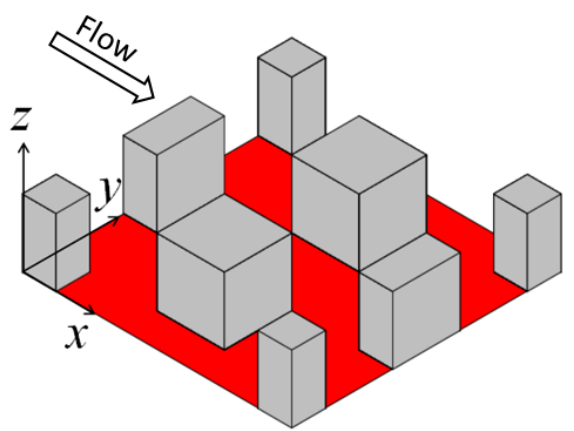

Fig. 1 Sketch of 3-D view of computational domain. All the bottom surface between cubes is heated or cooled.

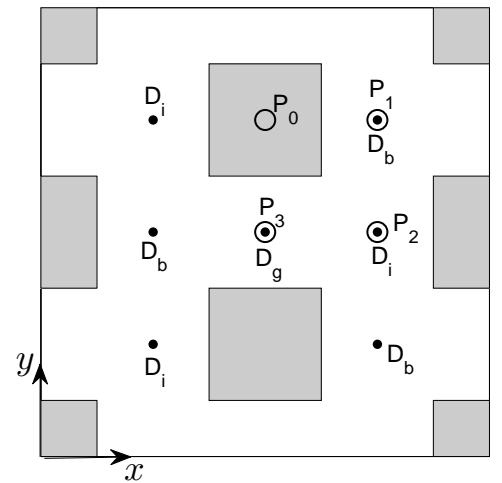

Fig. 2 Plan view of computational domain. The four typical locations, $\mathrm{P}_{0-3}$ are identified by 'circles' and data at 'dots' $\mathrm{D}_{\mathrm{b}}, \mathrm{D}_{\mathrm{i}}, \mathrm{D}_{\mathrm{g}}$ are used for quadrant analysis in Sect. 3.5.

The filtered governing equation for temperature is

$$
\frac{\partial T}{\partial t}+\frac{\partial u_{j} T}{\partial x_{j}}=\frac{\partial}{\partial x_{j}}\left(\left(k_{s}+k_{m}\right) \frac{\partial T}{\partial x_{j}}\right),
$$

where $T$ is the resolved-scale temperature. $k_{s}$ is the subgrid turbulent diffusivity and is given by $v_{s} / \operatorname{Pr}_{s}$, where $v_{s}$ is the subgrid viscosity and $\operatorname{Pr}_{s}$ is the subgrid Prandtl number whose value was set to $0.9 . k_{m}$ is the molecular diffusivity and is defined as $v / P r_{m}$, where $P r_{m}$ is the molecular Prandtl number whose value was set to 0.71 in our computations. Periodic boundary conditions were specified in the streamwise and spanwise directions. The stable stratification in the computational domain was obtained by specifying a negative heat flux at the bottom surface and the same was set to enter through the top surface. Similarly, the unstable stratification was obtained by specifying a positive heat flux at the bottom surface of the computational domain and the same was set to leave through the top surface. These computations were done on a domain size of $L_{x} \times L_{y} \times L_{z}=4 h \times 4 h \times 6 h$ (D4), where $h=0.2 \mathrm{~m}$ is the cube height. Whilst this domain is probably too small to capture adequately the long streamwise rolls known to exist in the outer flow, earlier work has demonstrated that it is sufficient for domain-independent mean flow fields, particularly within the canopy region. For example, based on two-point measurements on an array of the same configuration, Castro et al. (2006) showed that the integral length scales are constant in the region $2 \leq z / h \leq 4$ and are $3 h, 0.8 h$ and $h$ in $x, y$ and $z$ directions respectively. Also, the DNS study by Coceal et al. (2006) showed that the mean flow field is independent of the domain sizes $4 h \times 4 h \times 4 h, 8 h \times 8 h \times 4 h$ and $4 h \times 4 h \times 6 h$. 3-D and plan views of the computational domain are shown in Figs. 1 and 2 respectively. A finite volume approach was followed to discretize the flow and temperature equations. The monotone advection and reconstruction scheme (STAR-CD, 2007) with a blending factor of 0.9 was used for the spatial convective terms and the central difference scheme was used for the spatial diffusive terms of (1) and (3). A second-order 
backward implicit scheme was used for discretizing the time-dependent term. The computational domain D4 consisted of hexahedral cells and the grid resolution was $h / 16$. The driving force was the constant streamwise pressure gradient in Eq. (1) on every cell and is given by

$$
\frac{\partial\langle P\rangle}{\partial x}=\frac{\rho u_{\tau}^{2}}{L_{z}}
$$

where $u_{\tau}$ is the total wall friction velocity. The Reynolds number $\left(R e_{\tau}\right)$ based on the total wall friction velocity and $h$ was approximately 1200 . The Reynolds number (Re) based on $h$ and the streamwise velocity at $h$ varied from 3000 to 5000. The initial duration of most of the simulations was approximately 200 $e_{t}$ where $e_{t}=h / u_{\tau}$ is the eddy turn-over time. The averaging duration varied from $200 e_{t}$ to $400 e_{t}$ depending on how rapidly the shear and dispersive stresses converged. All the computations were carried out using STAR-CD version 4.14 (STAR-CD, 2007).

Sensitivity tests were done by conducting a further four independent sets of computations. They are

1. D4T - constant temperature instead of constant heat flux was specified on the top and bottom surfaces of the computational domain D4.

2. D4S - As an alternative means of achieving steady state for energy in the computational domain, constant heat sink (source) for unstable (stable) stratification was specified in all computational cells in D4 instead of a constant heat flux boundary condition on the top surface.

3. D16 - the domain size was $8 h \times 8 h \times 10 h$ with constant heat flux on the top and bottom surfaces of the domain. The vertical resolution varied geometrically from $h / 64$ at $z=0$ to $h / 16$ at the building height i.e $z=h$, and in the remaining parts of the domain $h / 16$ was used.

4. D64 - the domain size was $16 h \times 16 h \times 10 h$ with constant heat flux on the top and bottom surfaces of the domain. A uniform resolution of $h / 16$ was set throughout the domain.

A summary of all computations is given in Table 1.

\section{Results}

The first objective stated at the end of the Sect. 1 is addressed by determining the drag and heat transfer coefficients, displacement height $d$ and roughness length $z_{0}$ for various $R i_{\tau}$ in Sect. 3.1 and 3.2 respectively. By analysing the Reynolds stress anisotropy map, spatial correlations, quadrant and octant events for stable, neutral and unstable cases, the second objective is addressed and the details are presented in the latter subsections.

\subsection{Drag and heat transfer coefficients}

The degree of thermal heating or cooling can be characterized by the Richardson number $R i_{\tau}$ defined as

$$
R i_{\tau}=\frac{g h\left(T_{b}-T_{z=0}\right)}{T_{b} u_{\tau}^{2}}
$$




\begin{tabular}{|c|c|c|c|c|c|c|}
\hline $\begin{array}{l}\text { Type of } \\
\text { instability }\end{array}$ & Domain size & $\begin{array}{c}\text { Type of thermal } \\
\text { boundary condition }\end{array}$ & $\begin{array}{c}q_{z=0}\left(\mathrm{Wm}^{-} 2\right) \text { or } \\
T_{z=0}(\mathrm{~K})\end{array}$ & $R i_{\tau}$ & $C_{d}$ & $C_{h}$ \\
\hline \multirow{7}{*}{ Stable } & \multirow{7}{*}{$\begin{array}{c}4 h \times 4 h \times 6 h \\
\text { (D4) }\end{array}$} & \multirow{7}{*}{$\begin{array}{l}\text { constant } \\
\text { heat flux }\end{array}$} & -3 & 0.8775 & 0.0739 & 0.0066 \\
\hline & & & -8 & 2.5099 & 0.0645 & 0.0057 \\
\hline & & & -10 & 3.1986 & 0.0662 & 0.0057 \\
\hline & & & -12.5 & 4.1042 & 0.0628 & 0.0054 \\
\hline & & & -15 & 4.9978 & 0.0618 & 0.0053 \\
\hline & & & -18 & 6.1868 & 0.0569 & 0.0049 \\
\hline & & & -25 & 8.9943 & 0.0552 & 0.0046 \\
\hline \multirow{7}{*}{ Unstable } & \multirow{7}{*}{$\begin{array}{c}4 h \times 4 h \times 6 h \\
(\mathrm{D} 4)\end{array}$} & \multirow{7}{*}{$\begin{array}{l}\text { constant } \\
\text { heat flux }\end{array}$} & 1 & -0.2737 & 0.0758 & 0.0071 \\
\hline & & & 3 & -0.7909 & 0.0779 & 0.0075 \\
\hline & & & 8 & -2.0472 & 0.0812 & 0.0079 \\
\hline & & & 12.5 & -3.0969 & 0.0856 & 0.0084 \\
\hline & & & 25 & -6.0259 & 0.0959 & 0.0091 \\
\hline & & & 50 & -11.6382 & 0.1158 & 0.0104 \\
\hline & & & 100 & -22.3893 & 0.1552 & 0.0125 \\
\hline \multirow{4}{*}{ Unstable } & \multirow{4}{*}{$\begin{array}{c}4 h \times 4 h \times 6 h \\
(\mathrm{D} 4 \mathrm{~T})\end{array}$} & \multirow{4}{*}{$\begin{array}{c}\text { constant } \\
\text { temperature }\end{array}$} & 293.35 & -0.2703 & 0.0765 & 0.0072 \\
\hline & & & 294 & -0.7788 & 0.0796 & 0.0074 \\
\hline & & & 297 & -3.1465 & 0.0868 & 0.0084 \\
\hline & & & 307 & -11.0886 & 0.1155 & 0.011 \\
\hline \multirow{2}{*}{ Unstable $^{\mathrm{a}}$} & \multirow{4}{*}{$\begin{array}{c}4 h \times 4 h \times 6 h \\
(\mathrm{D} 4 \mathrm{~S})\end{array}$} & & 3 & -0.7343 & 0.0761 & 0.008 \\
\hline & & constant & 8 & -1.9416 & 0.0811 & 0.0083 \\
\hline \multirow{2}{*}{ Stable ${ }^{b}$} & & heat flux & -8 & 2.0722 & 0.0688 & 0.0072 \\
\hline & & & -12.5 & 3.2955 & 0.064 & 0.0068 \\
\hline \multirow{2}{*}{ Unstable } & $8 h \times 8 h \times 10 h$ & constant & 8 & -1.504 & 0.0791 & 0.0106 \\
\hline & (D16) & heat flux & 25 & -4.1804 & 0.0862 & 0.0125 \\
\hline \multirow{2}{*}{ Unstable } & $16 h \times 16 h \times 10 h$ & constant & 3 & -0.75 & 0.0814 & 0.0081 \\
\hline & (D64) & heat flux & 8 & -1.9387 & 0.0817 & 0.0084 \\
\hline \multirow{3}{*}{ Neutral } & D4 & & & & 0.0759 & \\
\hline & D16 & - & - & 0 & 0.0762 & - \\
\hline & D64 & & & & 0.0816 & \\
\hline
\end{tabular}

Table 1 Summary of computational cases.

a - To establish a steady state for energy, constant heat sink is specified throughout the domain.

$\mathrm{b}$ - To establish a steady state for energy, constant heat source is specified throughout the domain.

where $g$ is the acceleration due to gravity and $T_{b}$ is the bulk temperature, which is the average temperature over the whole domain. It is to be noted that $R i_{\tau}$ is not known $a$ priori, but is an outcome of the computation that depends on the specified boundary conditions. The values of $R i_{\tau}$ along with the resulting coefficients are listed in Table 1. Instead of using the bulk or gradient Richardson numbers to represent the degree of thermal stratification, a frictional Richardson number is used here because the former two depend on domain size and particularly good accuracy in determination of the flux gradients, respectively. In the conventional definition of $R i_{\tau}$, which is often used in (open) channel flows (e.g. Armenio and Sarkar, 2002; Dong and Lu, 2005; GarcíaVillalba and del Álamo, 2011), the density or temperature difference between the two surfaces and channel half height are used. This definition is modified here for two reasons: (i) because a roughness height is a more appropriate characteristic length and (ii) similar to the bulk velocity, temperature distribution inside the domain also depends on domain height. Therefore, the temperature difference between the ground surface and bulk temperature instead of that at the top surface is used. 
The thermal impact on the surrounding flow can be quantified using drag $\left(C_{d}\right)$ and heat transfer $\left(C_{h}\right)$ coefficients defined here as

$$
C_{d}=\frac{u_{\tau}^{2}}{u_{z=h}^{2}}
$$

$$
C_{h}=\frac{q_{z=0}}{c_{p} \rho u_{z=h}\left(T_{b}-T_{z=0}\right)}
$$

where $c_{p}$ is the specific heat capacity at constant pressure and $q_{z=0}$ is the heat flux at the ground surface. Note that when constant heat flux was specified on the bottom surface, $T_{z=0}$ is the spatially and temporally averaged non-uniform surface temperature. Similarly when constant temperature was specified on the ground surface, $q_{z=0}$ is the spatially and temporally averaged non-uniform surface heat flux. The procedure for obtaining $T_{z=0}$ or $q_{z=0}$ (STAR-CD, 2007) was as follows:

$$
T^{+}= \begin{cases}P r_{m} z^{+} & \text {if } z^{+} \leq z_{T}^{+} \\ \left(P r_{s}+P r_{m}\right)\left(u^{+}+P\right) & \text { if } z^{+}>z_{T}^{+}\end{cases}
$$

where

$$
T^{+}=\frac{c_{p} \rho\left(T_{z=0}-T_{z_{1}}\right) u_{*}}{q_{z=0}}
$$

and

$$
u^{+}= \begin{cases}z^{+} & \text {if } z^{+} \leq z_{u}^{+} \\ \frac{1}{\kappa} \ln \left(E z^{+}\right) & \text {if } z^{+}>z_{u}^{+}\end{cases}
$$

Here $z^{+}=z_{1} u_{*} / v, T_{z_{1}}$ is the temperature at the near-wall grid point, $z_{1}$ is the distance from the wall to the centre of the near-wall grid point, $u_{*}$ is the near-wall friction velocity determined by Spalding's law (Shih et al., 1999) and $P$ is the sub-layer resistance factor (Jayatilleka, 1969). $z_{u}^{+}$and $z_{T}^{+}$satisfy the following equations:

$$
\begin{aligned}
z_{u}^{+}-\frac{1}{\kappa}\left(E z_{u}^{+}\right) & =0 \\
P r_{m} z_{T}^{+}-\left(P r_{s}+P r_{m}\right)\left[\frac{1}{\kappa} \ln \left(E z_{T}^{+}\right)+P\right] & =0
\end{aligned}
$$

where $E$ is an empirical coefficient whose value was set to 9 . It was observed in our computations that most of the near-wall grid points lie within the viscous sublayer.

For the basic case, D4, Figs. 3a and b show an increase in $C_{d}$ and $C_{h}$ as the thermal stratification changed from stable to unstable. For $R i_{\tau}<0$, a similar increasing trend was also found by Cheng and Liu (2011) and Kanda et al. (2007) in 2-D street canyons and the COSMO (Comprehensive Outdoor Scale Model) experiments respectively. Such an increase is due to a gradual increase in the strength of the turbulence motions, as illustrated by the data in Fig. 8a (discussed later). In comparison with the flow over smooth terrain, stability effects on the flow over a rough surface are likely to be lower because of the dominant influence of the mechanical turbulence generated by the roughness elements. However, the assumption that urban flows may be considered as neutral or nearly neutral in urban dispersion models (Britter and Hanna, 2003) is probably invalid, as the results presented above suggest that stratification effects are not negligible. 

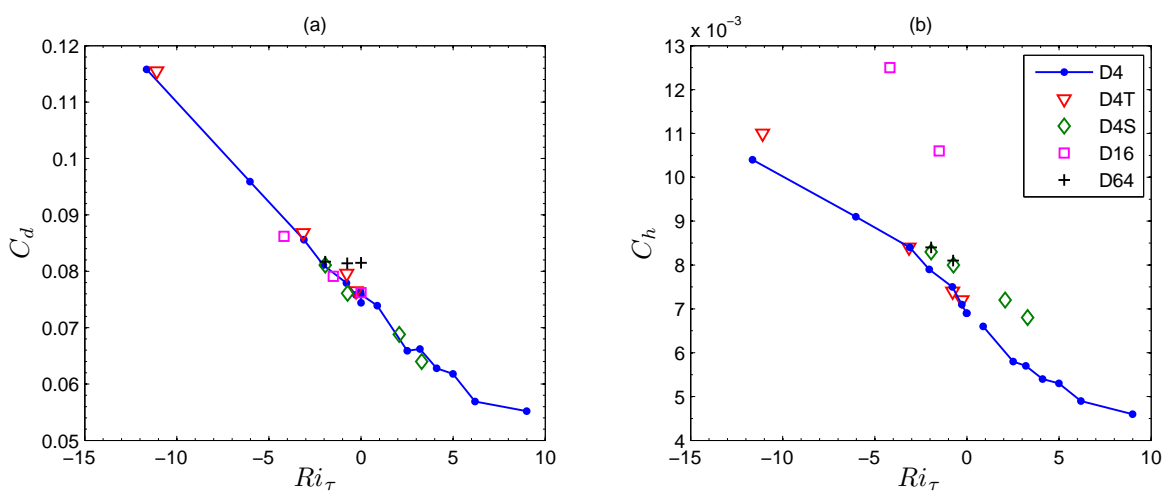

Fig. 3 Variation of (a) $C_{d}$ and (b) $C_{h}$ with $R i_{\tau}$. For the legend details, see Table 1.

\subsubsection{Sensitivity checks}

As mentioned in Sect. 2, the sensitivity tests were done by performing computations on different domain sizes and grid resolution. In Figs. $3 \mathrm{a}$ and $\mathrm{b}$, the values of $C_{d}$ and $C_{h}$ from D16 and D64 are also shown. It can be observed that $C_{d}$ from D4 and D16 are in good agreement. Both D4 and D16 show gradual increase in $C_{d}$ with $R i_{\tau}$, while the drag coefficient from D64 remains constant as $R i_{\tau}$ decreases from 0 to -1.94 but is anyway quite close to the results from the smaller domains. Figure 3(b) shows that the values of $C_{h}$ from D64 are approximately 7\% larger and those from D16 are approximately $41 \%$ larger than D4. The significant increase seen in D16 can perhaps be partly attributed to domain size effects but, much more importantly, is a direct result of the much finer resolution near the ground surface. Although we have shown that it is necessary to employ fine resolution near the surface to predict scalar transfer coefficients very accurately (Boppana et al., 2010), to save on expensive computational time (which would be particularly demanding for D64) an identical uniform resolution of $h / 16$ was enforced in all D4 and D64 cases. These computations show that $C_{d}$ is fairly insensitive to both domain size and resolution but the estimation of $C_{h}$ is indeed significantly affected by the mesh resolution. Therefore, the variation of $C_{h}$ with $R i_{\tau}$ shown here should be considered as a qualitative indicator only.

Figures $3 \mathrm{a}$ and $\mathrm{b}$ also show that the two types of thermal boundary conditions, i.e constant heat flux (D4) and constant temperature (D4T) on bottom and top surfaces of the computational domain, yield very similar values of $C_{d}$ and $C_{h}$. Even though a constant heat flux (temperature) boundary condition at the bottom of a rough wall yields a non-uniform distribution of temperature (heat flux) around the obstacles, this study confirms that the integral quantities are not significantly affected by the different physics at the ground surface.

To establish a steady state for energy, all D4 unstable (stable) computations had constant heat flux entering (leaving) through the ground surface and leaving (entering) through the top surface of the computational domain. But this can also be achieved by specifying constant sink (source) in all cells of the computational domain for unstable (stable) cases and these simulations are classified as D4S. The differences 

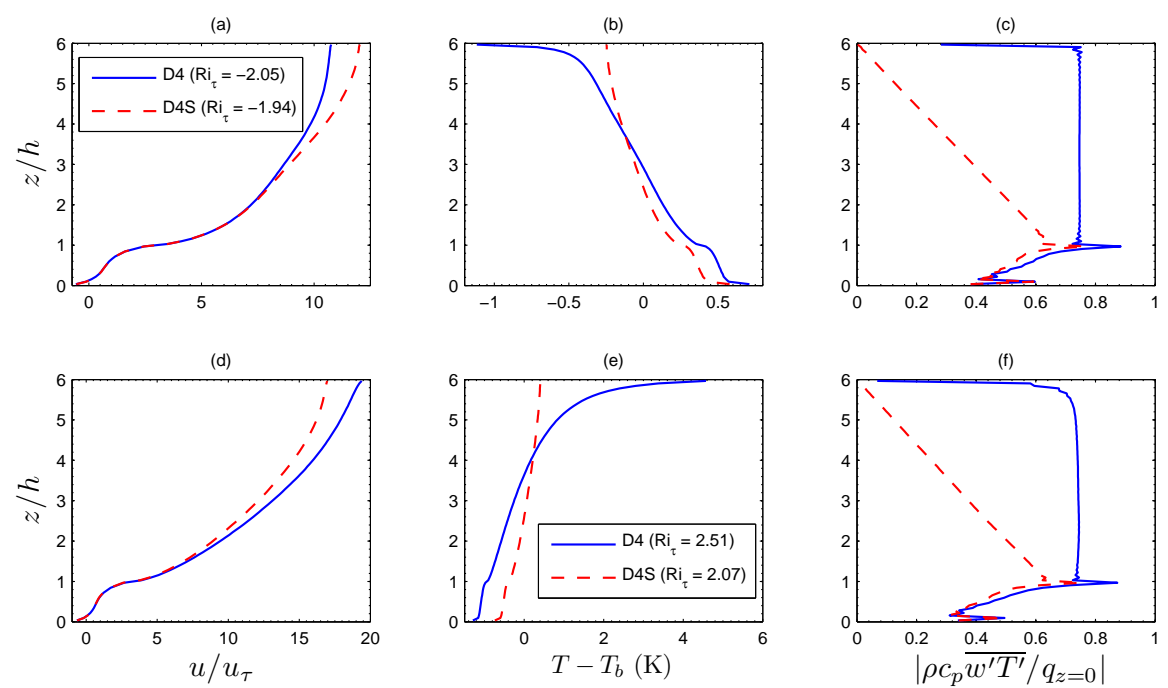

Fig. 4 Comparison of temporally and spatially averaged profiles of (a \& d) normalized streamwise velocity, (b \& e) temperature difference and (c \& f) normalized vertical turbulent heat flux for D4 and D4S. Top row: unstable, bottom row: stable. For the legend details, see Table 1.

in the vertical distribution of turbulent heat flux for D4 and D4S are shown in Fig. 4c for both stable and unstable cases. Figure 3a shows that the drag coefficient is not affected by the way in which steady state for energy is achieved, but the values of $C_{h}$ from D4S in Fig. $3 \mathrm{~b}$ are found to be $25 \%$ larger than in D4 for the stable case, while only $5 \%$ larger in the unstable case. The reason for such differences can be explained from the temporal and spatial mean of the temperature difference, shown in Fig. 4b. It can be observed that the temperature variation with height is very much dependent on the way in which steady state for energy is achieved. This in turn affects the flow field and can be seen in the spatial and temporal mean profiles of streamwise velocity in Fig. 4a. This brief numerical test suggests that heat transfer coefficients are sensitive to the way in which steady state for energy in the computational domain is realised. It would be quite challenging if not impossible to set up heat sinks or sources away from boundaries in a wind-tunnel experiment, and in any case such sources or sinks are not possible physically without the action of additional flow variables, like moisture content. Further analysis in this current study is therefore restricted to cases with constant heat flux boundary conditions on the top and bottom surfaces.

\subsubsection{A note on domain size and its influence on dispersive stresses}

Dispersive stresses, denoted by $\langle\widetilde{u} \widetilde{w}\rangle$ in the case of shear stress, arise due to spatial inhomogenities in the flow. Therefore, their presence is expected below the canopy but not far above. In the case of D4, the dispersive stresses above the canopy were very small. But in the case of D64, it was observed that the dispersive stresses above the canopy persisted even after a time average duration of $1000 e_{t}$. This is because D64 is conducive to the development of streamwise rolls that are larger in scale than 

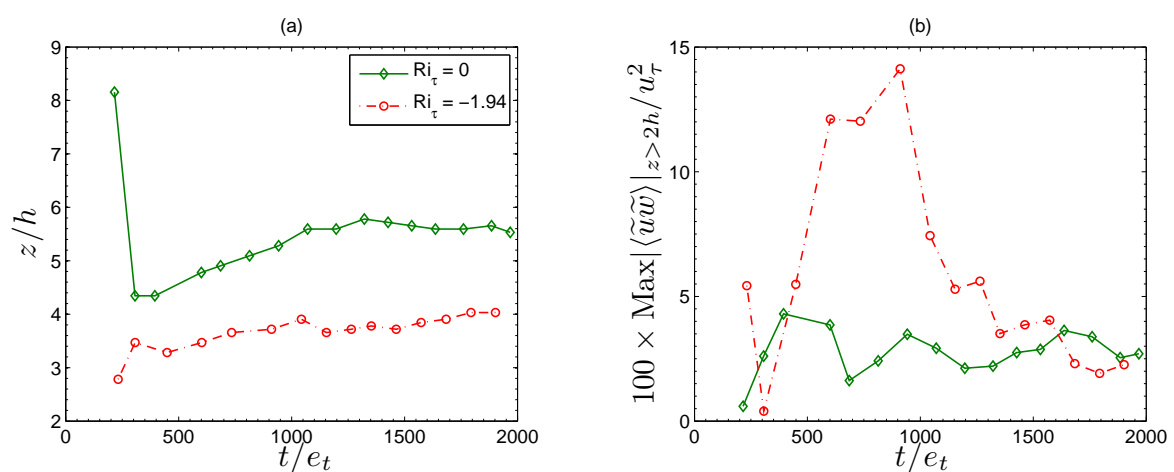

Fig. 5 (a) Location of maximum dispersive stress and (b) percentage variation of maximum dispersive stress with time mean duration. The initial duration for $R i_{\tau}=0$ and -1.94 are $200 e_{t}$ and $400 e_{t}$ respectively.

are allowed by domain D4. Such slow evolving mean longitudinal rolls are clearly shown in the DNS study of Coceal et al. (2006) for the neutral case. But it was also shown that for a sufficiently long averaging time i.e. $400 e_{t}$, these dispersive stresses above the canopy disappear. It was observed in the current study that the dispersive stresses above $z / h=2$ exhibit non-monotonic behaviour with increasing averaging time. This can be seen in Fig. 5b, where the percentage variation with averaging time of maximum dispersive stress for $z>2 h$ is shown. It can be observed that the maximum dispersive stress above the canopy appears to be converging to approximately $2.5 \%$ of the wall stress (or approximately $5 \%$ of the shear stress at that height) and the location at which it occurs is around $z / h=5.5$ and 4 for neutral and unstable cases respectively. In a systematic set of investigations conducted by Fishpool et al. (2009) in a turbulent channel flow at $R e_{\tau}=410$, it was observed that (i) the spanwise inhomogenities persisted even when the domain length was increased from $2 \pi \delta$ to $62 \delta$, where $2 \delta$ is the channel depth and (ii) these features remained, with a large magnitude, for time averaging in excess of $10 \delta / u_{\tau}$ (Fishpool et al., 2009, called $\delta / u_{\tau}$ the 'friction time scale'). Detailed investigations are being carried out on D64 to determine the averaging time required for the dispersive stresses to completely disappear (if they do) and the reason for their existence over long durations.

\subsection{Determination of pressure distribution, $d$ and $z_{0}$}

It was observed in Sect. 3.1 that the increase in the drag coefficient with decreasing $R i_{\tau}$ is correlated with an increase in the turbulent kinetic energy. More directly, however, it is the pressure difference between the windward and leeward sides of the cubes which determine the (form) drag. The vertical profiles of time- and laterallyaveraged pressure coefficients $\left(C_{p}\right)$ were obtained for various $R i_{\tau}$ and are shown in Fig. 6a. The pressure coefficient is defined as

$$
C_{p}=\frac{\left(p_{w}-p_{l}\right)}{\frac{1}{2} \rho u_{z=h}^{2}},
$$




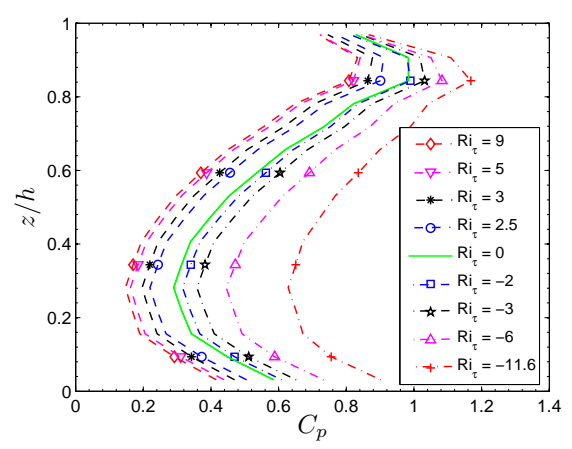

(a)

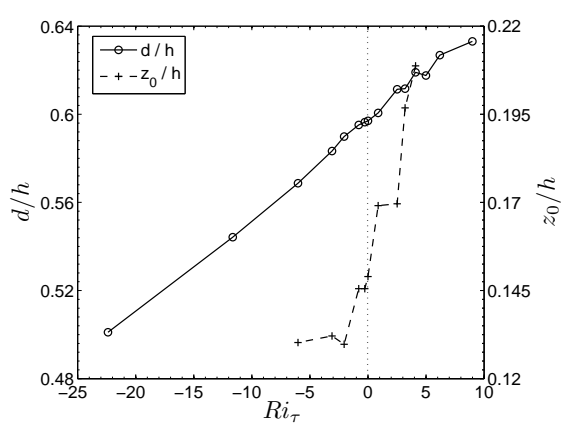

(b)

Fig. 6 Variation of (a) temporally and laterally averaged normalised pressure coefficient, and (b) mean displacement height and roughness length with $R i_{\tau}$.

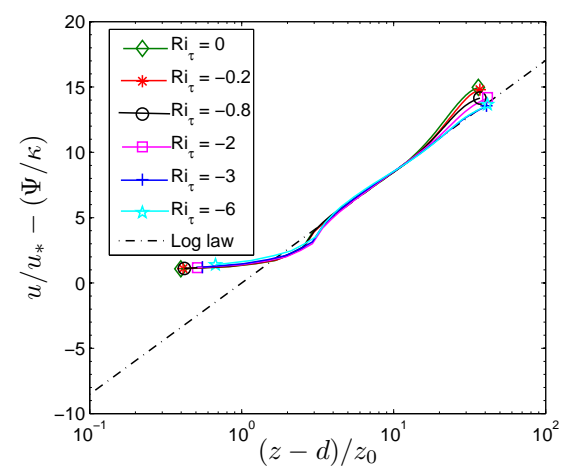

(a)

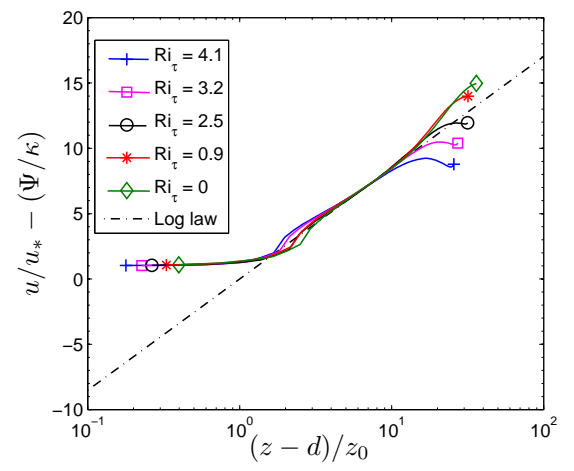

(b)

Fig. 7 Spatial- and temporal-averaged mean streamwise velocity profiles in log-linear form for various $R i_{\tau}$ (a) neutral and unstable flows (b) neutral and stable flows.

where $p_{w}$ and $p_{l}$ are the pressures on the windward and leeward faces of the cube respectively. It can be observed that there is a notable increase in the values of $C_{p}$ with ground heating and a slow decrease with ground cooling. The form drag, $C_{p d}$, can be obtained by integrating Eq. 13 with respect to $z$, and in all cases is approximately $85 \%$ of $C_{d}$. (The remaining drag component arises from frictional forces on the ground and the top and sides of the cubes, see Leonardi and Castro (2010) for a discussion on this point.)

The most sensible definition of the zero plane displacement height, $d$, is that it is the height at which the surface drag acts (Jackson, 1981). Assuming that frictional forces are negligible, this can be written (Coceal et al., 2006) as,

$$
d=\frac{\int_{z} z\left(p_{w}-p_{l}\right) \mathrm{dz}}{\int_{z}\left(p_{w}-p_{l}\right) \mathrm{dz}} .
$$

With the data shown in Fig. 6a this suggests that $d$ decreases with increase in heating, as confirmed in Fig. 6 b. Although the change only amounts to some $25 \%$ over the 
range of $R i_{\tau}$ covered, one would expect corresponding, but larger, changes in the roughness length $z_{0}$, which was indeed found to be the case and can be seen in Fig. 6b. The procedure of obtaining $z_{0}$ at various $R i_{\tau}$ is briefly described below.

The wind speed profile for non-neutral condition is given by (Stull, 2009):

$$
\frac{u}{u_{*}}=\frac{1}{\kappa}\left[\ln \left(\frac{z-d}{z_{0}}\right)+\Psi\left(\frac{z-d}{L}\right)\right],
$$

where $\kappa$ is von Kármán's constant and $L$ is the Obukhov length defined as

$$
L=-\frac{\left[{\overline{u^{\prime} w^{\prime}}}_{z=0}^{2}+{\overline{v^{\prime} w^{\prime}}}_{z=0}^{2}\right]^{3 / 4}}{\kappa\left(g / \bar{T}_{v}\right)\left(\overline{w^{\prime} T_{v}^{\prime}}\right)_{z=0}} \equiv-\frac{u_{*}^{3}}{\kappa\left(g / \bar{T}_{z=0}\right)\left(q_{z=0} / \rho c_{p}\right)} .
$$

Here primed quantities denote deviation from their respective mean values, $\bar{T}_{v}$ is mean virtual potential temperature and $\overline{w^{\prime} T_{v}^{\prime}}$ is the mean kinematic virtual potential temperature flux in the vertical direction. The stability function $\Psi((z-d) / L)$ is typically given as (Stull, 2009)

$$
\Psi\left(\frac{z-d}{L}\right)=\left\{\begin{array}{lr}
4.7(z-d) / L & \text { for } R i_{\tau}>0 \\
-2 \ln \left[\frac{1+\gamma}{2}\right]-\ln \left[\frac{1+\gamma^{2}}{2}\right]+2 \tan ^{-1}(\gamma)-\frac{\pi}{2} & \text { for } R i_{\tau}<0
\end{array}\right.
$$

where

$$
\gamma=\left[1-\zeta \frac{z-d}{L}\right]^{1 / 4} \text { where } \zeta=15
$$

In Eq. 15, $\Psi=0$ yields the standard logarithmic law for neutral (rough-wall) flow, with $u_{*}$ the surface friction velocity. (Note that the addition of the non-neutral term $(\Psi)$ in Eq. 15 breaks the usual monotonic correspondence between $z_{0}$ and $u_{*}$, so that for $R i_{\tau} \neq 0 z_{0}$ may rise when $u_{*}$ falls or vice versa.) For a pressure-driven channel flow Coceal et al. (2006) derived $u_{*}=u_{\tau} \sqrt{\left(1-d / L_{z}\right)}$ to account for the linear variation in shear stress from $z / h=d$ to $L_{z}$ which otherwise is constant in the surface layer of the atmospheric boundary layer. $\zeta$ in Eq. 18 is changed to 16 such that the resulting $\Psi$ agreed with that given in Table 1.1 of Kaimal and Finnigan (1994).

Using $d$ from Eq. 14, the values of $\kappa$ and $z_{0}$ are obtained as fitting parameters of Eq. 15 for neutral flow. The necessary value of $\kappa$ was found to be 0.27 , which is $34 \%$ lower than the classical value of 0.41. A similar discrepancy from the classical value was also reported by Cheng and Castro (2002), Coceal et al. (2007), Leonardi and Castro (2010) to name a few.

By fixing $\kappa$ as 0.27 and using the computed value of $d$ for each $R i_{\tau}, z_{0}$ was deduced by fitting the measured $u$ profile to Eq. 15 over a height range of $z / h=1.5$ to 2.5 - approximately chosen such that the variations of individual estimates of $z_{0}$ from the velocity at a specific height in this range was less than $10 \%$. However, for $R i_{\tau}<-6$ and $>4.1$, the variation of $z_{0}$ in the above mentioned range of $z / h$ exceeded $10 \%$ and hence these data are not included in Fig. $6 \mathrm{~b}$.

Figure 7 shows the vertical variation of spatial- and temporal-averaged velocity profiles for neutral, stable and unstable cases. It can be observed that the LES data is not incompatible with the log-linear form and that for increasing $\left|R i_{\tau}\right|$ the data appear to shift gradually to the right of the neutral case; this movement is found to be slightly stronger in stable flows. 

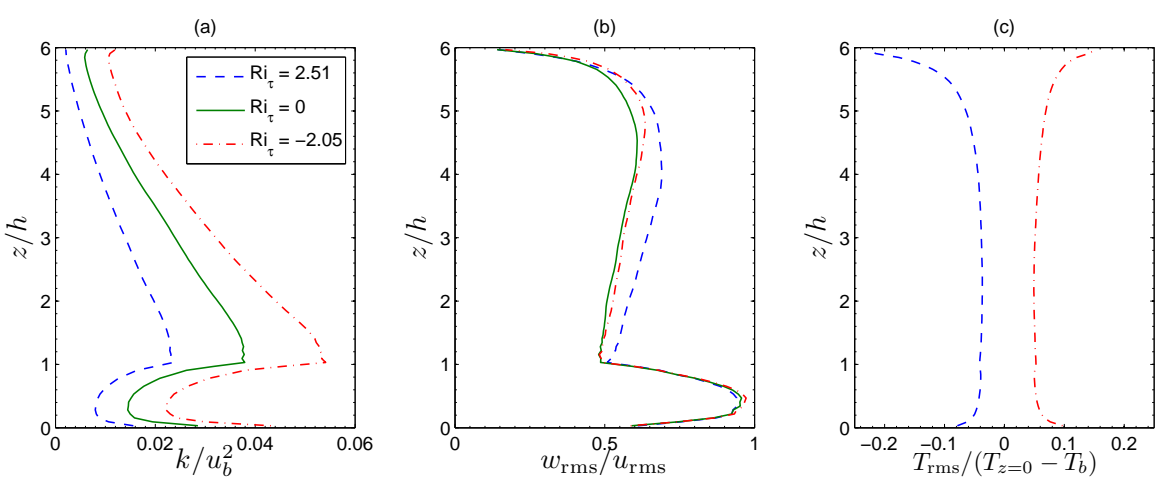

Fig. 8 Temporal and spatial mean of (a) turbulent kinetic energy normalized with bulk velocity, (b) ratio of vertical to streamwise Reynolds stresses and (c) normalized temperature fluctuations for $R i_{\tau} \approx-2,0$ and 2.5 .

\subsection{Turbulence level and Reynolds stresses anisotropy}

Some effects of thermal stratification on the turbulence field are shown in Fig. 8 for D4. Increase in the normalized turbulent kinetic energy with decrease in $R i_{\tau}$ is evident in Fig. 8a. The ratio of vertical to streamwise fluctuations in Fig. 8b is found to be nearly the same for neutral and unstable cases thus suggesting that this structural parameter is not affected by ground heating, at least within the range $0>R i_{\tau} \geq-2$. However, for the stable case at $R i_{\tau}=2.5$ the ratio is found to be slightly larger than in the neutral and unstable cases. This indicates that the turbulence structural characteristics of the stable case are different to those of neutral and unstable cases. Therefore, further exploration of turbulence structure have been carried out and are discussed in the following paragraphs. Figure 8(c) shows that the normalized temperature fluctuations are almost constant throughout the domain height, except near the bottom and top surfaces where the temperature gradients are inevitably strongest because of proximity to the imposed boundary conditions.

The anisotropy of the time mean Reynolds stresses is often used as an indicator of turbulence structure and this is shown using Lumley's anisotropy invariant map, AIM (Pope, 2011). Figure 9 shows AIM for various $R i_{\tau}$ and for four typical locations, as identified by Castro et al. (2006) and indicated as $\mathrm{P}_{0-3}$ in Fig. 2. The AIM is obtained from the second and third principle invariants of the stress tensor $b_{i j}, 6 \eta^{2}=-2 \mathrm{II}_{b}=$ $b_{i j} b_{j i}$ and $6 \xi^{3}=3 \mathrm{III}_{b}=b_{i j} b_{j k} b_{k i}$, where

$$
b_{i j}=\frac{\left\langle\overline{u_{i} u_{j}}\right\rangle}{2 k}-\frac{\delta_{i j}}{3} .
$$

The vertical axis $\eta$ of the AIM gives the magnitude of the anisotropy and the horizontal axis $\xi$ represents the shape of anisotropy (i.e. distinguishing qualitatively between 'rod-like' and 'disc' shaped turbulent eddies). The linear sides of the triangle originating from $(\xi, \eta)=(0,0)$ represent axisymmetric turbulence and the origin indicates isotropy. $\xi>0$ implies 'rod-like' shaped turbulence where two eigenvalues of the Reynolds stress tensor are smaller than the third one and $\xi<0$ refers to 'disc' 
(a) $\mathrm{P}_{0}$ (top)

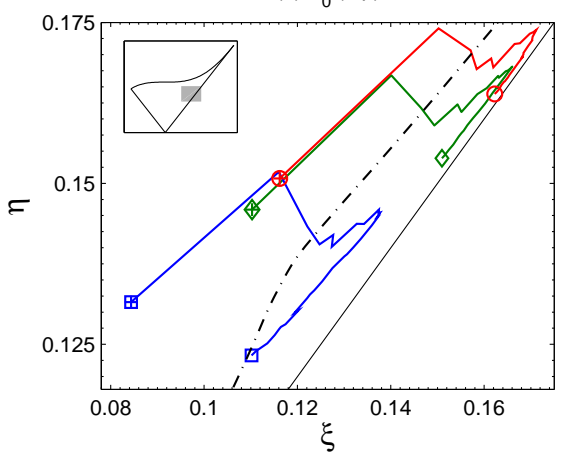

(c) $P_{2}$ (in front)

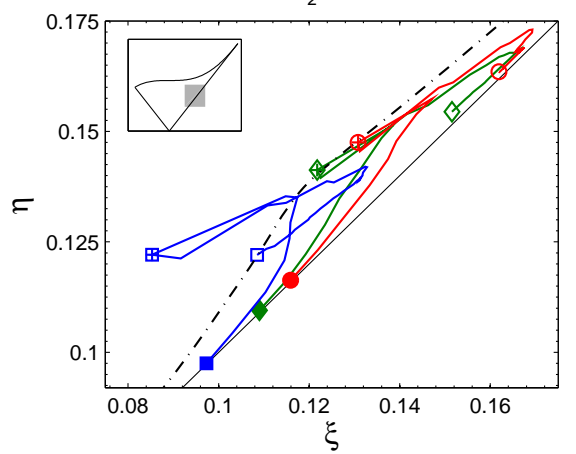

(b) $P_{1}$ (behind)

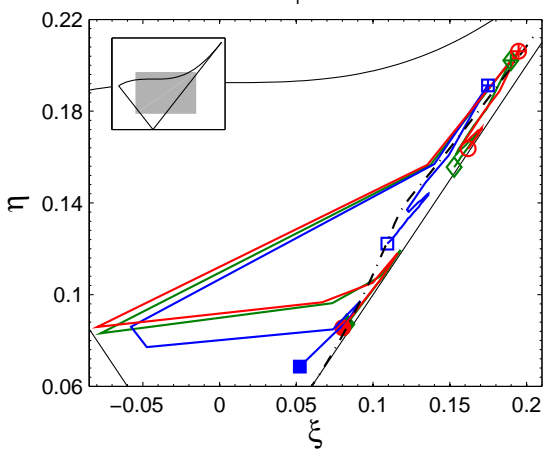

(d) $P_{3}$ (gap)

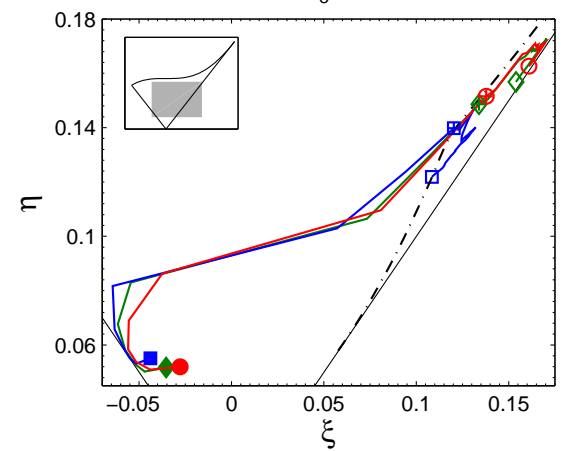

Fig. 9 AIM at typical locations for $R i_{\tau} \approx-2$ (circles), 0 (diamonds) and 2.5 (squares). For clarity, only the immediate regions occupied by the data are shown, with solid black lines indicating boundaries of the Lumley triangle where appropriate; the inset figures show these regions in a grey shade, in relation to the entire Lumley triangle. Filled symbols are at $z / h=0.5$, open symbols with an internal ' + ' are at $z / h=1$ and clear open symbols are at $z / h=3$. The dash-dot line near the right outline of the Lumley triangle is the logarithmic and core region data $(30 \leq z+\leq 180)$ from smooth wall turbulent channel flow with $R e_{\tau}$ $=180$ (Busse and Sandham, 2012); here the data approach $(\xi, \eta)=(0,0)$ with increasing distance from the wall.

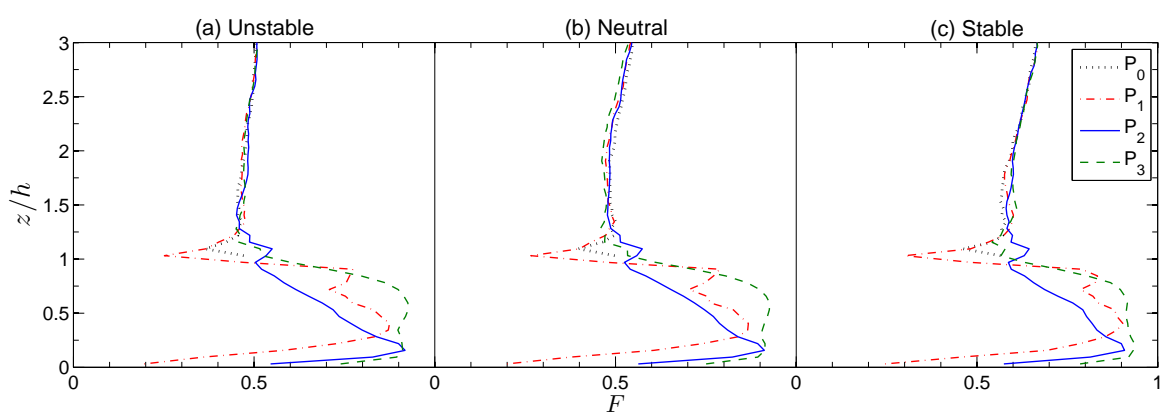

Fig. 10 The anisotropy function for (a) $R i_{\tau}=-2$ (unstable), (b) 0 (neutral) and (c) 2.5 (stable) cases at typical locations. 
shaped turbulence where two eigenvalues are greater than the third eigenvalue of the Reynolds stress tensor. The upper curve of the triangle represents two-component turbulence where one of the eigenvalues is zero.

For clarity, data within the AIM are shown only for $0.5 \leq z / h \leq 3$ in Fig. 9. The data shown at each typical location $\mathrm{P}_{i}$, where $i=0-3$, are temporal and spatial means at the four identical locations in the computational domain. Comparison of AIM data for $\mathrm{P}_{1}$ and $\mathrm{P}_{2}$ for $R i_{\tau}=0$ with the experimental values of Castro et al. (2006) show qualitative agreement (not shown here).

It is observed that the shapes of profiles for neutral and unstable cases are very similar and they differ mostly in the magnitude of anisotropy. The structure of the anisotropy for the stable case is found to be slightly different to that of neutral and unstable cases. At all four typical locations, the magnitude of anisotropy is found to be generally lower in a stable and higher in an unstable case, and this is very evident in the profiles at $\mathrm{P}_{0}$ and $\mathrm{P}_{2}$. For $z / h>1.2$ (i.e. in the log-linear region of the mean velocity profile), the profiles at all four locations are on or close to the right outline of the Lumley map just as they are in the log and core region of a smooth-wall turbulent channel flow at $R e_{\tau}=180$ (Busse and Sandham, 2012). This suggests an axisymmetric nature of turbulence with predominantly 'rod-like' shaped eddies. With increasing $z / h$ above the canopy the data tend to move towards the origin, just as they do in the smooth-wall channel flow. However, note that, unlike the data in the neutral and unstable cases that are very close to right outline of the Lumley map, stable case data are a little further away from the right boundary. Overall, we conclude that even with surface heating or cooling the turbulence structure in the log region (i.e. above the urban canopy) is not very different to that in the log region of flow over smooth surfaces. This indicates that for $z / h>1.2$, the turbulent structure is similar to that of smooth-wall boundary layer. The fact that in neutral flows urban-type roughness does not have a large effect on turbulence structure at least qualitatively within the log law has previously been noted by Coceal et al. (2006). Based on the field measurements, same observation was made by Roth et al. (2013) and this is conceptually shown in the Fig. 6 of their article. It is interesting that the same seems to be true for cases of moderate ground heating or cooling. The data suggest that changes become apparent soonest for stable cases but, in any case, one would not expect the same conclusion to hold if $R i_{\tau}$ were to increase to very large magnitudes.

As expected, the shapes of profiles at the lower heights (between $z / h=0.5$ and 1.2) differ significantly at the various locations. At $P_{1}$ and with $z / h$ increasing from 0.5 , the turbulence structure becomes more 'disc' shaped, which could be due to the recirculation region, and again changes back to 'rod-like' shape as the profile reaches the canopy height. With increasing $z / h$ at $\mathrm{P}_{2}$, the turbulence structure appears to drift gradually away from the 'rod-like' shape and revert back to this shape for $z / h>1$. At $\mathrm{P}_{3}$, where the mean flow field experiences 'channeling' effects, the presence of side-walls appears to encourage the turbulence structure to be more 'disc' shaped, which is counter-intuitive.

A direct measure of the degree of isotropy in the turbulence is provided by the parameter $F=1+I I_{b}+27 I_{1} ; F=0$ and 1 represents two-component and isotropic turbulence respectively. The values of this parameter at the four typical locations and for various $R i_{\tau}$ are shown in Fig. 10. As expected, the values of $F$ vary considerably 
below the canopy, but not above where the flow is essentially homogeneous in $x$ and $y$. Owing to the strong three-dimensional effects, the turbulence below the canopy becomes increasingly isotropic as $z$ approaches zero, especially at $\mathrm{P}_{1}$ and $\mathrm{P}_{3}$, until very close to the wall when of course eddies are strongly constrained vertically. Such high values of $F$ were also observed in the wind-tunnel experiments of Castro et al. (2006) for the neutral case. Perhaps surprisingly, the values of $F$ below the canopy are found to be almost same for stable, neutral and unstable cases. This must be due to the very high turbulence intensities caused by shear and the wake of the cubes, which are not strongly reduced by surface heating or cooling. But above the canopy, the stable case shows slightly larger values of $F$ compared to neutral and unstable cases.

The above analysis was also carried out for case D64 with $R i_{\tau}=0$ and -2 ; the corresponding figures (not shown here) show qualitatively similar behaviour to that for D4. Differences were most evident above the canopy, no doubt because of the non-zero dispersive stresses there.

\subsection{Spatial correlations}

In order to determine the influence of thermal stratification on the integral length scales of the turbulent structures, two-point velocity correlations were computed. The spatial correlation for streamwise velocity in the streamwise direction is given by (e.g. Castro et al., 2006)

$$
R_{u u}(\Delta x)=\frac{\overline{u^{\prime}(x) u^{\prime}(x+\Delta x)}}{\sigma_{u}^{\prime}(x) \sigma_{u}^{\prime}(x+\Delta x)} .
$$

The two-point correlation of vertical velocity in the vertical direction is obtained by replacing $u$ and $x$ in Eq. (20) with $w$ and $z$ respectively. Figure 11 shows these computed correlations for D4; the streamwise spatial correlations are shown at $z / h=$ 1.28 and the vertical spatial correlations are obtained by specifying $z / h=1.53$ as a fixed reference. It is observed in this figure that $R_{u u}(\Delta x)$ does not tend to zero at $\Delta x$ $=2$, which is half of the streamwise domain length. This suggests that the domain length is not sufficient to capture the longest eddy structures. Nonetheless, we can make some deductions from the data.

Figure 11 shows that $R_{u u}(\Delta x)$ for the stable case is lower than that of the neutral and heated cases. The streamwise integral length scale has clearly been significantly reduced by ground cooling, but appears not to be influenced by ground heating. The reason for such a strong influence on streamwise length scales by stable stratification is not yet completely understood, although it is well known that stability generally weakens turbulence fields. The profiles of $R_{w w}(\Delta z)$ indicate that the vertical integral length scales are marginally increased and decreased by ground heating and cooling respectively. This is expected because the size of the vertical structures is enhanced by thermal plumes due to buoyancy in an unstable case and reduced in the case of stable stratification. These spatial correlations suggest that the turbulent structures are smaller in stable stratification when compared to neutral and unstable cases. As smaller structures tend to be more isotropic, this observation is consistent with the 
(a)

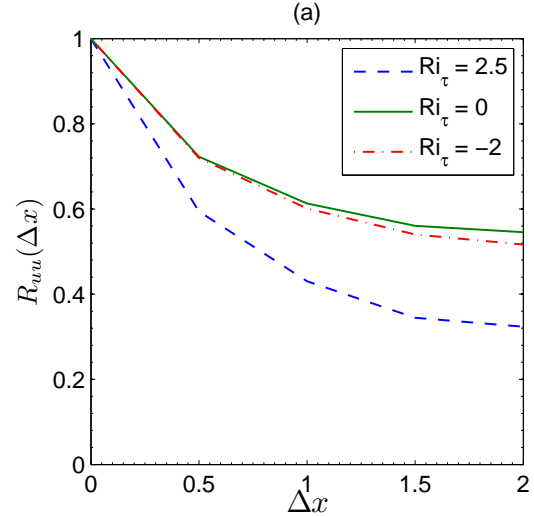

(b)

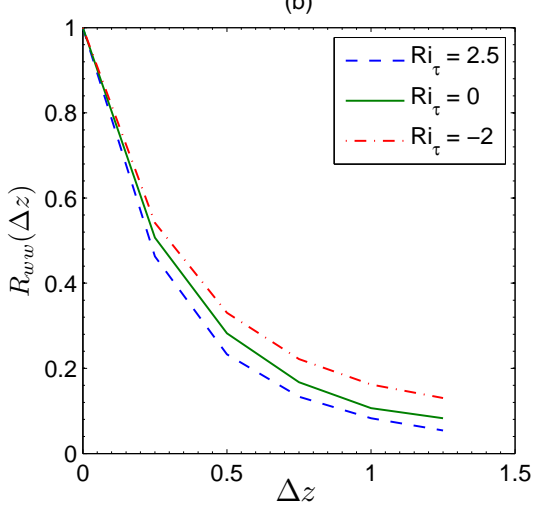

Fig. 11 Spatial correlations of (a) $u$ in $x$ direction and (b) $w$ in $z$ direction for different $R i_{\tau}$.

implications of the AIM discussed in Sect. 3.3. The spatial correlations from D64 for neutral and unstable cases are, incidentally, found to be similar to those of D4.

\subsection{Quadrant and Octant Analysis}

The occurrence and contribution of various intermittent events to the transfer of momentum and heat is often deduced using quadrant analysis. According to this, the events are classified as follows

$$
\begin{array}{lll}
\text { Q1 : } & u^{\prime}>0, w^{\prime}>0 ; & \theta^{\prime}>0, w^{\prime}>0 \\
\text { Q2 }: & u^{\prime}<0, w^{\prime}>0 ; & \theta^{\prime}<0, w^{\prime}>0 \\
\text { Q3 : } & u^{\prime}<0, w^{\prime}<0 ; & \theta^{\prime}<0, w^{\prime}<0 \\
\text { Q4: } & u^{\prime}>0, w^{\prime}<0 ; & \theta^{\prime}>0, w^{\prime}<0
\end{array}
$$

where primed quantities refer to fluctuating values (about their respective time-means). In the case of momentum flux, 'Q2' refers to movement of low-speed fluid in the upward direction (referred as 'ejections') and 'Q4' refers to movement of high-speed fluid in the downward direction (referred as 'sweeps'). In the case of stable stratification, 'Q2' refers to those events where cold fluid moves in the upward direction (termed as 'updrafts') and 'Q4' refers to those events where hot fluid moves in the downward direction (termed as 'downdrafts'). In the case of unstable stratification, 'Q1' refers to 'updrafts' where hot fluid is ejected and 'Q3' refers to 'downdrafts' where cold fluid moves in the downward direction. The difference in the frequency of occurrence of sweeps and ejections, and downdrafts and updrafts, and their proportional contribution to total momentum and heat fluxes (often referred to as 'flux fraction', but here we use the term 'strength') are shown in Fig. 12. The method used to obtain the frequency and strength of momentum and heat flux for various events is explained in detail in Boppana et al. (2013). The values shown in Figs. 12a, d are obtained using a time average of $330 e_{t}$ and a spatial average of data at all the seven locations shown as dots in Fig. 2 and identified as $D_{b}, D_{i}$ and $D_{g}$. The values shown 

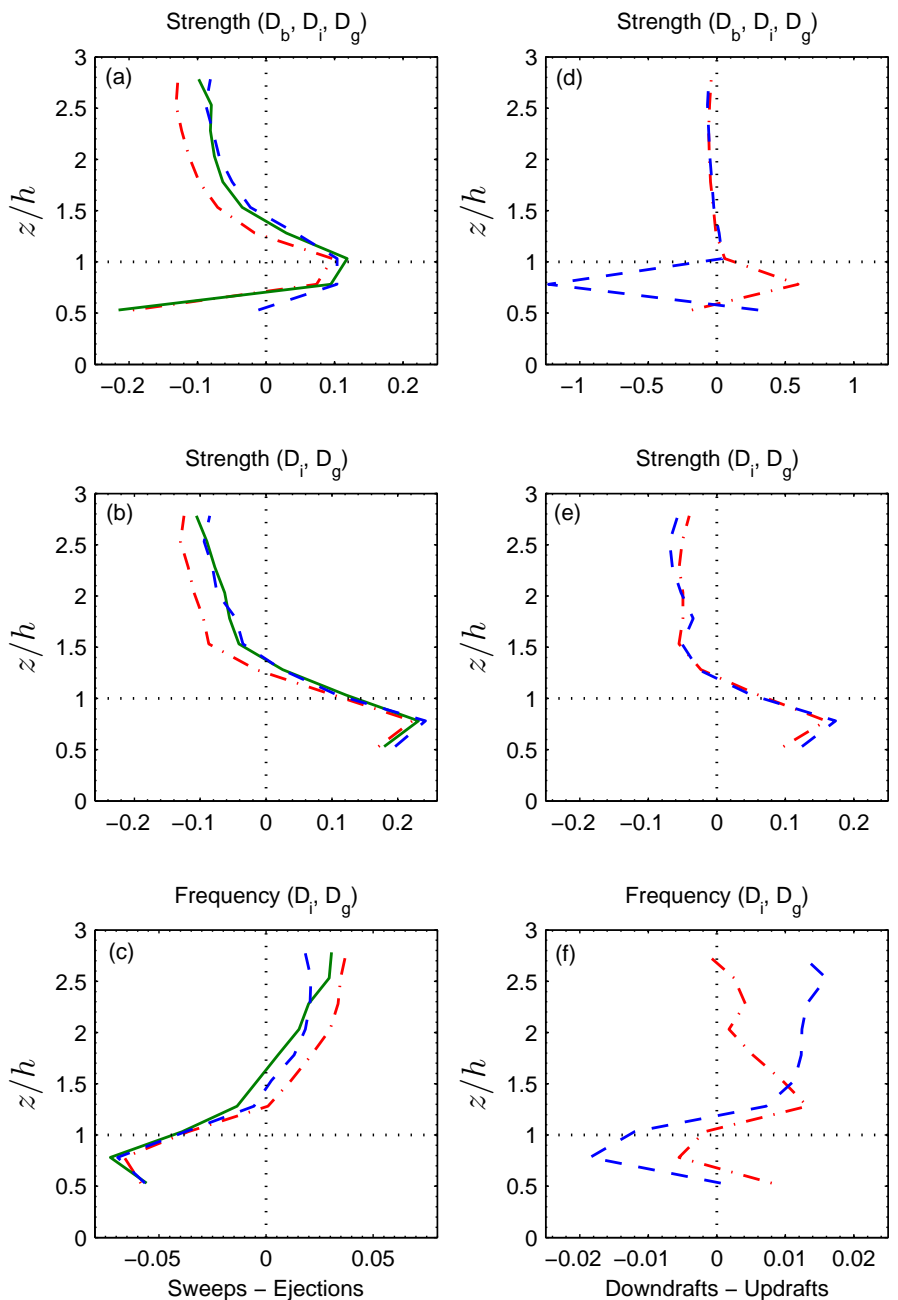

Fig. 12 Left column: differences in the contributions to momentum flux by sweeps (Q4) and ejections (Q2) (a), (b) and the difference in their frequency of occurrence (c); right column: differences in the contributions to heat flux by downdrafts (Q4 - stable, Q3 - unstable) and updrafts (Q2 - stable, Q1 unstable) (d), (e), and the difference in their frequency of occurrence (f). Dash-dot lines: unstable $\left(R i_{\tau}=\right.$ $-2)$; solid lines: neutral $\left(R i_{\tau}=0\right)$; dash lines: stable $\left(R i_{\tau}=2.5\right)$. The time and spatial average of data from $\mathrm{D}_{b}, \mathrm{D}_{i}$ and $\mathrm{D}_{g}$ locations (shown in Fig. 2) are used in (a) and (d), and the average of data from $\mathrm{D}_{i}$ and $\mathrm{D}_{g}$ are used in (b), (c), (e) and (f).

in Figs. 12b, c, e and $\mathrm{f}$ are from a time and spatial average of the four locations $\mathrm{D}_{\mathrm{i}}$ and $\mathrm{D}_{\mathrm{g}}$ which do not lie in the recirculating regions immediately behind the cubes.

The time and spatial average of data from all seven locations shows that ejections are stronger above the canopy (Fig. 12a), but below the canopy ejections dominate at $z / h \approx 0.5$ whilst, for $0.5 \leq z / h \leq 1$, sweeps contribute more to the momentum flux. Such a non-monotonic behaviour below the canopy is a result of the strong influence 
of the recirculating region in the wake of the cubes. This influence is also observed in the strength of events contributing to heat flux (Fig. 12d). As suggested in the DNS study by Coceal et al. (2007), it is instructive to obtain the temporal and spatial mean from all locations in the computational domain. But as the available data here is limited to seven locations, the data from the three locations behind the cubes $\left(D_{b}\right)$ have been excluded in some of the results shown so as to prevent the strong influence from the recirculation region biasing the results of the quadrant analysis. Figure $12 \mathrm{~b}$ then shows that momentum flux is dominated by sweeps below the canopy, which is consistent with the observations made in the DNS study. (Including the three 'behind cube' profile locations destroys that consistency.) Further analysis will therefore be based on the time and spatial average data from the $D_{i}$ and $D_{g}$ locations only, shown in Figs. 12b, c, e and f.

Below the canopy, the strength and frequency of momentum flux events in Figs. $12 \mathrm{~b}, \mathrm{c}$ are found to be the same for unstable, neutral and stable cases. This implies that the mechanical turbulence generated by the roughness elements has a much stronger influence than the local thermal stratification. Further above the canopy, thermal stratification, especially for the unstable case, appears to have a notable effect as the strength of ejections and the frequency of sweeps is enhanced. In the field study of Christen et al. (2007), point measurements from a tower in an urban street canyon showed qualitatively similar behavior except that the strength of ejections begins to dominate sweeps at $z / z_{h}=1.9$ for an unstable case and at $z / z_{h}=2.5$ for a near-neutral case, whereas sweeps dominated throughout the measurement height i.e. $0.5 \leq z / z_{h} \leq 2.5$ in the stable case ( $z_{h}$ is an average building height). The reason for these minor differences between field experiments and LES could be partly attributed to the urban morphometry, different $R i_{\tau}$, prevailing meteorological conditions (e.g. large-scale turbulent motions (Michioka et al., 2011) and wind direction) in the field.

Similar to the momentum flux contributions in stable and unstable cases, downdrafts contribute more to the heat flux below the canopy and updrafts are stronger above the canopy. Figure 12f suggests the reverse behaviour in the frequency of events. The field study of Christen et al. (2007) showed similar behaviour in the strength of events, but the stratification effects were found to be strong above the canopy unlike this study, probably for reasons similar to those mentioned above.

The same analysis was carried out on time series data corresponding to a duration of $2000 e_{t}$ and from eight locations situated in front of the cubes in D64. The strength and frequency of events were found to be qualitatively very similar to those described above for the D4 domain.

From the above analysis, it is understood that for both stable and unstable cases, above the canopy ejections and updrafts contribute more to the momentum flux and heat flux respectively, whereas within the canopy sweeps and downdrafts dominate. Sweeps and downdrafts occur more often above the canopy, whilst ejections and updrafts are more frequent within the canopy. But it is not immediately clear if the updraft (downdraft) and ejection (sweep) events are correlated. Inagaki et al. (2012) showed that the horizontal distribution of ejection and sweep events at the building height is similar to the distribution of updraft and downdraft events suggesting that these events might be correlated. To determine this quantitatively, octant analysis (as used by Dupont and Patton, 2012, on a vegetation canopy) has been conducted. Based 


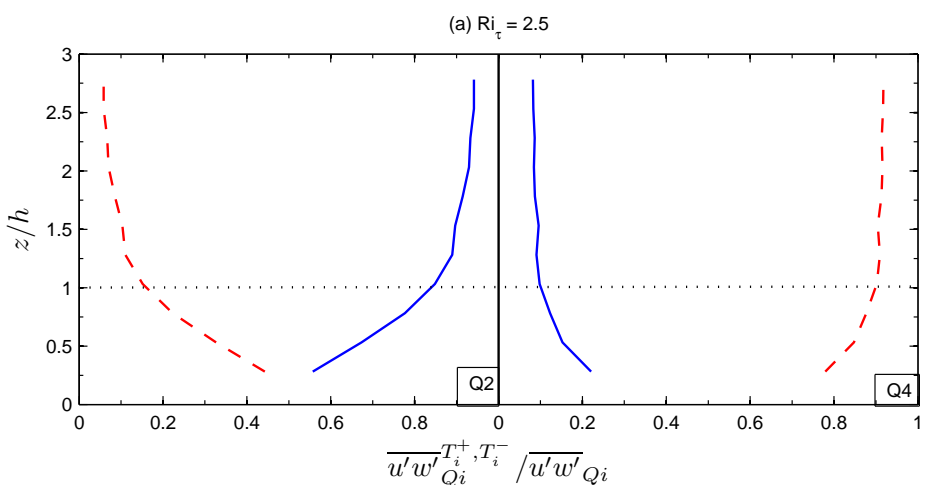

(b) $\mathrm{Ri}_{\tau}=-2$

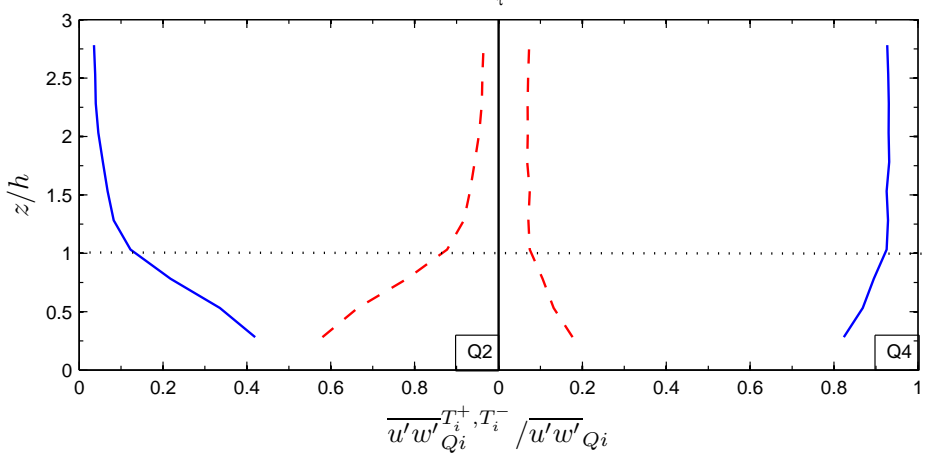

Fig. 13 Momentum flux associated with positive (dashed line) and negative (positive line) temperature fluctuations in quadrants 2 and 4 for (a) stable and (b) unstable cases. The dotted line indicates the canopy top.

on the sign of temperature fluctuations, the momentum flux from a quadrant ' $Q i$ ' is split further such that

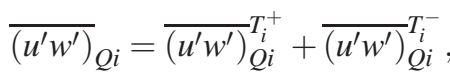

where $T_{i}^{+}$and $T_{i}^{-}$correspond to positive and negative temperature fluctuations respectively in the quadrant $Q i$. The two right hand terms in the above equation are normalized with their respective quadrant momentum fluxes and are shown in Fig. 13. For both stable and unstable cases the 'updrafts' contribution to the momentum flux is found to be larger in 'Q2' and the 'downdrafts' contribution is found to be larger for 'Q4'. This suggests that updrafts (downdrafts) and ejections (sweeps) are well correlated, which implies at least some degree of similarity in momentum and heat transport for such flows. 


\section{Conclusions}

The effects of local thermal stratification on the atmospheric flow in and above urban canopies have been investigated by conducting large-eddy simulations on flow past an array of staggered cubes, with the ground surface subjected to uniform cooling or heating. The global thermal influences have been quantified by computing drag and heat transfer coefficients. With increase in ground surface heating, characterised by $-23<R i_{\tau}<-0.2$, a gradual increase in $C_{d}$ and $C_{h}$ was observed. Specification of either constant heat flux or constant temperature boundary condition on the ground surface yielded similar values of $C_{d}$ and $C_{h}$, despite the different physics of flow and heat very close to the ground surface. With increase in ground surface cooling, i.e. $0<R i_{\tau}<9$, a gradual decline in $C_{d}$ and $C_{h}$ was observed. The steady increase in $C_{d}$ and $C_{h}$ with decrease in $R i_{\tau}$ is linked with an increase in turbulent kinetic energy due to buoyancy. The sensitivity tests included computations with different domain sizes, grid resolution and means of achieving the steady state for energy in the computational domain. These showed that $C_{d}$ was relatively insensitive to all these, but the estimates of $C_{h}$ were found to be very sensitive to resolution in the near-wall region, not surprisingly.

The structure of the turbulence for $R i_{\tau}=-2,0$ and 2.5 was then quantitatively analysed by exploring the Reynolds stresses, spatial correlations and the results of quadrant and octant analyses. The turbulence intensity was found to be significantly affected by ground heating and cooling. However, the anisotropy invariant maps implied that the shape of the turbulent structures remained very similar for neutral and unstable cases, but differed slightly in the stable case. From the two-point spatial correlations it was observed that the turbulent integral length scales of the structures are reduced in both streamwise and vertical directions by stable stratification when compared to the neutral case; only the vertical integral length scale was found to be increased by ground heating. The quadrant analysis showed that ground heating (cooling) enhances (reduces) the contribution of ejections to momentum flux above the canopy whereas the contribution of updrafts and downdrafts to heat flux are found to be very similar. Octant analysis showed that the strength of ejections (sweeps) and updrafts (downdrafts) are well correlated, thereby suggesting that the transport mechanisms of momentum and heat flux are similar above the canopy, probably because of the prevailing large-scale structures although no attempt has yet been made to study the correlated spectral content between ejections and updrafts in order to delineate scale effects.

This study has shed some light on the effects of local thermal stratification on the aerodynamic coefficients and turbulent structure of flow over an idealised urban canopy. It would be useful to know whether the general conclusions outlined above apply also to different kinds of roughness morphology, and to what extent they are affected by differential surface heating arising for example from radiation. Coupled with the present results, this might then be a further step towards understanding and modelling the pollutant dispersion in significantly non-neutral urban boundary layers.

Acknowledgements This project is funded by the Natural Environment Research Council, through its National Centre for Atmospheric Sciences, Grant No. R8/H12/38. All computations have been done using 
CD-adapco's STAR-CD code on Iridis (University's supercomputer) and HECToR (U.K's supercomputer). We thank the CD-adapco's staff Fred Mendonca for his continuous support.

\section{References}

Allegrini J, Dorer V, Carmeliet J (2013) Wind tunnel measurements of buoyant flows in street canyons. Build Environ 59:315-326

Armenio V, Sarkar S (2002) An investigation of stably stratified turbulent channel flow using large-eddy simulation. J Fluid Mech 459:1-42

Boppana VBL, Xie ZT, Castro IP (2010) Large-eddy simulation of dispersion from surface sources in arrays of obstacles. Boundary-Layer Meteorol 135:433-454

Boppana VBL, Xie ZT, Castro IP (2013) Large-eddy simulation of heat transfer from a single cube mounted on a very rough wall. Boundary-Layer Meteorol 147:347368

Britter RE, Hanna SR (2003) Flow and dispersion in urban areas. Annu Rev Fluid Mech 35:469-496

Busse A, Sandham ND (2012) Parametric forcing approach to rough-wall turbulent channel flow. J Fluid Mech 712:169-202

Cai X (2012) Effects of differential wall heating in street canyons on dispersion and ventilation characteristics of a passive scalar. Atmos Environ 51:268-277

Castro IP, Cheng H, Reynolds R (2006) Turbulence over urban-type roughness: deductions from wind-tunnel measurements. Boundary-Layer Meteorol 118:109_ 131

Cheng H, Castro IP (2002) Near wall flow over urban-like roughness. BoundaryLayer Meteorol 104:229-259

Cheng WC, Liu CH (2011) Effect of unstable thermal stratification on the atmospheric boundary layer above urban street canyons by large-eddy simulation. The $14^{\text {th }}$ International conference on harmonisation within atmospheric dispersion modelling for regulatory purposes (HARMO14), Kos, Greece, 2-6 October

Christen A, van Gorsel E, Vogt R (2007) Coherent structures in urban roughness sublayer turbulence. Int J Climatol 27:1955-1968

Coceal O, Thomas TG, Castro IP, Belcher SE (2006) Mean flow and turbulence statistics over groups of urban-like cubical obstacles. Boundary-Layer Meteorol 121:491-519

Coceal O, Dobre A, Thomas TG, Belcher SE (2007) Structure of turbulent flow over regular arrays of cubical roughness. J Fluid Mech 589:375-409

Dong YH, Lu XY (2005) Direct numerical simulation of stably and unstably stratified turbulent open channel flows. Acta Mech 177:115-136

Dupont S, Patton EG (2012) Momentum and scalar transport within a vegetation canopy following atmospheric stability and seasonal canopy changes: the chats experiment. Atmos Chem Phys 12:5913-5935

Fishpool GM, Lardeau S, Leschziner MA (2009) Persistent non-homogeneous features in periodic channel-flow simulations. Flow Turbulence Combust 83:323-342

Gao W, Shaw RH, Pawu KT (1989) Observation of organized structure in turbulent flow within and above a forest canopy. Boundary Layer Meteorol 47:349-377 
García-Villalba M, del Álamo J (2011) Turbulence modification by stable stratification in channel flow. Phys Fluids 23:045,104-22

Huizhi L, Bin L, Fengrong Z, Boyin Z, Jianguo S (2003) A laboratory model for the flow in urban street canyons induced by bottom heating. Adv Atmos Sciences 20:554-564

Inagaki A, Castillo MCL, Yamashita Y, Kanda M, Takimoto H (2012) Large-eddy simulation of coherent flow structures within a cubical canopy. Boundary-Layer Meteorol 142:207-222

Jackson PS (1981) On the displacement height in the logarithmic velocity profile. J Fluid Mech 111:15-25

Jayatilleka CL (1969) The influence of prandtl number and surface rouhness on the resistance of the laminar sub-layer to momentum and heat transfer. Progress in heat and mass transfer 1:193-330

Kaimal JC, Finnigan JJ (1994) Atmospheric Boundary Layer Flows: Their Structure and Measurement. Oxford University Press, 304 pp

Kanda M, Kanega M, Kawai T, Moriwaki R (2007) Roughness lengths for momentum and heat derived from outdoor urban scale models. J Applied Meteorol and Climatol 46:1067-1079

Kim JJ, Baik JJ (1999) A numerical study of thermal effects on flow and pollutant dispersion in urban street canyons. J App Meteorol 38:1249-1261

Kovar-Panskus A, Moulinneuf L, Savory E, Abdelqari A, Sini JF, Rosant JM, Robins A, Toy N (2002) A wind tunnel investigation of the influence of solar-induced wallheating on the flow regime within a simulated urban street canyon. Water, Air, and Soil Pollution: Focus 2:555-571

Leonardi S, Castro IP (2010) Channel flow over large cube roughness: a direct numerical simulation study. J Fluid Mech 651:519-539

Li D, Bou-Zeid E (2011) Coherent structures and the dissimilarity of turbulent transport of momentum and scalars in the unstable atmospheric surface layer. Boundary-Layer Meteorol 140:243-262

Michioka T, Sato A, Sada K (2011) Wind-tunnel experiments for gas dispersion in an atmospheric boundary layer with large-scale turbulent motion. Boundary-Layer Meteorol 141:35-51

Park SB, Baik JJ, Raasch S, Letzel MO (2012) A large-eddy simulation study of thermal effects on turbulent flow and dispersion in and above a street canyon. $\mathrm{J}$ Appl Meteorol and Climatol 51:829-841

Pascheke F, Barlow JF, Robins A (2008) Wind-tunnel modelling of dispersion from a scalar area source in urban-like roughness. Boundary-Layer Meteorol 126:103124

Pope SB (2011) Turbulent flows. Cambridge University Press, 774 pp

Richards K, Schatzmann M, Leitl B (2006) Wind tunnel experiments modelling the thermal effects within the vicinity of a single block building with leeward wall heating. J Wind Eng Ind Aerodyn 94:621-636

Roth M, Inagaki A, Sugawara H, Kanda M (2013) Small-scale spatial variability of turbulent statistics, (co)spectra and turbulent kinetic energy measure over a regular array of cube roughness. Environ Fluid Mech DOI 10.1007/s10652-013-9322-3 
Shih TH, Povinelli LA, Liu NS, Potapczuk MG, Lumley JL (1999) A generalized wall function. Tech. rep., National Aeronautics and Space Administration

STAR-CD (2007) CD-adapco's CCM methodology, STAR-CD Version 4.04, 328 pp Stull RB (2009) An introduction to Boundary-Layer Meteorology. Springer, 674 pp Uehara K, Murakami S, Oikawa S, Wakamatsu S (2000) Wind tunnel experiments on how thermal stratification affects flow in and above urban street canyons. Atmos Environ 34:1553-1562

Xie ZT, Hayden P, Wood CR (2013) Large-eddy simulation of approaching-flow stratification on dispersion over arrays of buildings. Atmos Environ 71:64-74 